\title{
Il Partito Comunista Italiano e De Sanctis negli anni Cinquanta. Classe operaia ed egemonia nazionale ${ }^{1}$
}

\author{
Umberto Carpi \\ Università di Pisa \\ umberto.carpi@alice.it
}

\begin{abstract}
Nel secondo dopoguerra il Partito Comunista Italiano, sotto la guida di Palmiro Togliatti e alla luce dei Quaderni scritti in carcere da Antonio Gramsci (in particolare le note sul Risorgimento, sulla funzione degli intellettuali, sul concetto di egemonia) imposta una grande battaglia per la conquista dell'egemonia culturale. Il primo obiettivo è quello di combattere la prevalente influenza di Benedetto Croce e della sua interpretazione del Risorgimento, sostituendole appunto quella di Gramsci e facendo della classe operaia e del suo partito il vero erede della miglior tradizione laico-democratica del Risorgimento stesso, la tradizione di Bertrando Spaventa, di Francesco De Sanctis, di Antonio Labriola. L'oggetto di più aspra contesa fra liberali e marxisti, dalla stampa quotidiana alle riviste accademiche, diventa negli anni Cinquanta Francesco De Sanctis, secondo il dilemma diventato classico De Sanctis-Croce o De Sanctis-Gramsci? Strettamente legata, anzi propedeutica a questa è la discussione sul filosofo marxista Antonio Labriola. Una vera e propria 'battaglia delle idee' cui partecipano tutti, politici e intellettuali.
\end{abstract}

Parole chiavi: De Sanctis; Labriola; Croce; Gramsci; Risorgimento.

\section{Abstract}

In the second post-war period the Italian Communist Party, under the guidance of Palmiro Togliatti and in the light of the Quaderni written in prison by Antonio Gramsci (in particular the notes about the Risorgimento, the role of the intellectuals, and his concept of hegemony) poses a great battle for the conquest of cultural hegemony. The main purpose was to challenge the prevalent influence of Benedetto Croce and his interpretation of the Risorgimento, replacing precisely that of Gramsci and taking the working class and his party as the true heritage of the best lay-democratic tradition of the Risorgimento itself, the tradition of Bertrando Spaventa, of Francesco De Sanctis, and Antonio Labriola. The subject of this bitter confrontation between liberals and Marxists, to be found in the daily

1. La relazione che ho presentato in sunto l'11 dicembre 2008 è venuta via via assumendo, nella stesura, le dimensioni ampie di un libro (anche fittamente documentario). Qui perciò mi restringo a presentare alcuni paragrafi, particolarmente significativi di quel che nella relazione orale riassunsi. 
papers and in academic journals, becomes in the Fifties Francesco De Sanctis, according to the classic dilemma De Sanctis-Croce o De Sanctis-Gramsci?

Closely related to it, albeit occurring before this, is the discussion by the Marxist philosopher Antonio Labriola. A true and peculiar 'battle of ideas' in which all politicians and intellectuals participated.

Keywords: De Sanctis; Labriola; Croce; Gramsci; Risorgimento.

1. Col senno del poi si può ben dire che molte lacerazioni del fatale 1956 erano già latenti e che lo 'choc Stalin' dell'anno successivo funzionò da detonatore per micce già accese: né sembri allotrio questo cenno in un contesto desanctisiano, sol che si rifletta come il numero di «Società» 1953 specialmente dedicato a De Sanctis si aprisse con un ampio saggio commemorativo di Gastone Manacorda sull'Umanesimo di Stalin. Ebbene (fatta salva l'apologia di Stalin adesso irricevibile in quei termini, ma bisogna tener conto che nel 1953 tutti — anche i più accaniti avversari e magari nei termini più aspricommemorarono non un mostro, bensì un gigante della politica, e comunque oggi si richiederebbe maggior oggettività di giudizio storico), per capire quale equilibrio dai comunisti italiani si tentasse nel segno di $\mathrm{Gramsci}^{2}$ fra recupero della tradizione nazionale di cultura progressiva a guida borghese e inserimento nel processo mondiale di rivoluzione proletaria a guida sovietica e dunque per capire lo spirito di un'operazione filodesanctisiana a seguire un 'umanesimo di Stalin'; per capire anche quanto questa linea politica fosse temuta efficace dagli ambienti liberal-crociani, al punto di indurli a fondare nel 1954 una rivista - «Nord e Sud»— precipuamente destinata a contrastarla, serve rileggere le parole stesse del Manacorda. Parole tanto più significative perché uscite dalla penna del direttore della testata, storico del movimento operaio di indiscusso valore e autentico intellet tuale di raccordo (un po' come Carlo Salinari in campo letterario, Valentino Gerratana in campo filosofico, Massimo Aloisi in campo scientifico, ma Manacorda forse con minor rigidità di applicazione che non gli altri) fra Partito e mondo della cultura:

Il Croce agli inizi della sua vita intellettuale introdusse insieme con il Labriola lo studio del marxismo in Italia. Se egli fosse veramente d'esempio, se coloro che si considerano suoi discepoli avessero verso il nuovo e il vitale un interesse pari a quello che animava agli inizi il loro maestro, essi dovrebbero volgersi con pari attenzione allo studio degli sviluppi odierni del marxismo, che sono in Lenin e in Stalin, invece di ricalcare l'anticomunismo monotono e dogmatico degli ultimi anni del maestro, in nulla differente da quello che da più di un secolo usano, con scarso successo, i gesuiti per tenere $\mathrm{a}$ bada $\mathrm{i}$ liberali. Nel numero precedente di questa rivista, commemorando brevemen-

2. Esemplare e quasi didascalico già nel titolo, da questo punto di vista, l'articolo di Franco FERRI (uno degli storici coinvolti nell'edizione Einaudi di De Sanctis, per la quale curò nel 1960 Il Mezzogiorno e lo Stato unitario) su "Questione meridionale e unità nazionale in Gramsci», Rinascita, IX, 1, gennaio 1952, p. 6-10. 
te la morte di Benedetto Croce, scrivevamo che ad un approfondito colloquio critico con la sua opera occorre oggi presentarsi ricchi dell'esperienza della storia del mondo in questi ultimi trentacinque anni. Questa esperienza ha nell'opera di Stalin la sua forma più elevata. Se è vero, come è vero, che noi abbiamo bisogno, in questo momento della storia del nostro paese, di riaffermare la forza creativa della scienza contro il ritorno dell'oscurantismo, di difendere lo spirito critico contro le reinvoluzioni verso principi d'autorità variamente camuffati o addirittura neppure camuffati, di fondare una nuova moralità sociale, e di garantire la nostra stessa unità culturale nazionale dalle insidie che la minacciano [n.b., corsivo non nel testo], non possiamo ignorare che le armi per questa lotta sono state affilate là dove una lotta per molti aspetti affine è stata combattuta e vinta. ${ }^{3}$

Nel 1955, ripeto, quel che sarebbe successo nel 1956 non lo si sapeva: si sapeva bene, invece, quel che era successo nel 1948, con la sconfitta elettorale e il sostanziale isolamento del blocco di sinistra, il trionfo di un blocco moderato nel quale i partiti di democrazia laica si erano aggregati al grande partito cattolico in funzione anticomunista e antisocialista. D'altro dunque c'era bisogno che della storia e della prospettiva di un movimento operaio e popolare a sé e per sé: il suo punto di vista, certo, ma per una diversa storia generale della società italiana. Sono puntualmente del 1950 - in quest'ottica politico-storiografica- due fondamentali interventi di Togliatti, sul piano della storia la rivalutazione dell'operato a inizio secolo di Giovanni Giolitti per le sue aperture al Partito Socialista lette come disponibilità all'inserimento di quel Partito nel governo del Paese; sul piano della prospettiva, la riproposizione del celebre discorso 1946 su Ceto medio e Emilia rossa (il titolo dice tutto), di autentica svolta nella determinazione delle alleanze sociali. ${ }^{4} \mathrm{Dal}$ punto di vista culturale, nel $1948 \mathrm{fu}$ avviata in forma di volumi tematici la diffusione dei quaderni scritti in carcere da Gramsci: né li pubblicava l'editoria di partito, bensì la solita casa editrice Einaudi, torinese come l'Ordine Nuovo di Gramsci e di Togliatti però espressione della più raffinata borghesia laica e progressista, diretta discendenza dal grande economista liberale Einaudi Luigi, allora presidente della neonata Repubblica.

3. Nel testo si allude al redazionale (ma credo scritto dal Manacorda) «La morte di Benedetto Croce», Società, VIII, 4, dicembre 1952. Subito dopo la morte di Croce comparvero su «Rinascita» due interventi piuttosto severi, che segnalo per la particolare pertinenza al nostro tema, di C. SalinarI, "Benedetto Croce critico", Rinascita, IX, 11 novembre 1952, p. 621-25 («La cultura italiana chiude per sempre oggi il capitolo Benedetto Croce e ne apre uno nuovo: quello di Antonio Gramsci») e di M. AliCATA, "Benedetto Croce e il Mezzogiorno», ibid, IX, 12 dicembre 1952, p. 680-84 («'indifferenza, anzi il disprezzo del Croce per la presenza nella storia anche delle 'classi subalterne' toccano, quando egli volge lo sguardo al Mezzogiorno, il loro culmine, così acuta è in lui evidentemente la volontà che gli intellettuali meridionali non s'accostino alla realtà economica e sociale delle loro regioni e mantengano la loro 'pratica', come egli stesso ammonisce alla fine della Storia del Regno di Napoli, "dentro le accademie"»).

4. P. Togliatti, Discorso su Giolitti, Roma: 1950 e ID., Ceto medio e Emilia rossa, Roma, s.d. [1950, ma pronunciato nel 1946 a Reggio Emilia]. 
Da notare che la prima informazione-presentazione dei mitici 'quaderni' era stata affidata nientemeno che a Luigi Russo, proprio il crociano e desanctisiano Russo, il quale ne aveva fatto l'oggetto d'un celebre discorso (1947, decimo anniversario della morte di Gramsci, Antonio Gramsci e l'educazione democratica in Italia) alla Scuola Normale Superiore di Pisa, della quale era direttore $^{5}$ : non fu un caso che da quell'incarico il Russo venisse di lì a poco bruscamente rimosso, con un intervento censorio di inusuale durezza. Comunque, questa scelta di sottrarre i quaderni del carcere ad una chiusa circolazione partitica e di presentarne le riflessioni sulla storia e sulla società italiana riaggregate in saggi specifici sugli intellettuali, sul Risorgimento, su Benedetto Croce, su Machiavelli come elaborati da un grande politico-intellettuale nazionale piuttosto che dall'antico teorico dei consigli di fabbrica e dal dirigente comunista contribuì al successo clamoroso del loro impatto sulla cultura italiana anche non partiticamente orientata.

Molti in seguito hanno storto il naso, né solo per motivi filologici, di fronte a questa scelta editoriale di tematizzare, ma Togliatti aveva colto nel segno, e comunque ottenuto il suo scopo 'egemonico'. Era prima di tutto sul piano culturale infatti, per rompere l'isolamento e creare le condizioni di una svolta politica, che occorreva soppiantare l'egemonia del sistema ideologico dominante, il quale peraltro nella scuola e fra gli intellettuali non era quello cattolico omogeneo alla Democrazia Cristiana partito di maggioranza bensì l'idealistico, la propaganda clericale della Chiesa e delle sue emanazioni tipo Azione Cattolica avendo funzione ed efficacia piuttosto pratica e agitatoria (emblematico il disprezzo laico del pur moderato Montale nelle Processioni del '49, col tanfo acre dei loro madonnari pellegrini): di questa tal quale subalternità culturale al liberalismo laico-borghese i settori più integralisticamente cattolici —non sempre necessariamente i più reazionari, basti pensare alle "Cronache sociali» di Dossetti- avvertivano il disagio ${ }^{6}$ : bisognava rovesciare insomma, o per meglio dire bisognava far slittare la lettura della storia nazionale, impostata da Croce in funzione dell'egemonia d'una classe borghese a vocazione nazionale, in una lettura di quella medesima storia in funzione dell'egemonia d'una

5. Antonio Gramsci e l'educazione democratica in Italia, poi dal Russo raccolto in De vera religione. Noterelle e schermaglie, 1943-1948, Torino: Einaudi 1948, p. 232-56.

6. Penso all'interessante gruppo cattolico integralista di Gianni Baget Bozzo — peraltro partito dalle Cronache sociali di Dossetti- raccolto intorno a Terza generazione. Altro discorso andrebbe fatto per i guppi cattolici influenzati dall'idealismo (Il Mulino) o dal marxismo (Il Dibattito Politico). Ma lo iato fra potere politico dei cattolici e loro incapacità di egemonia culturale veniva registrato come un fatto. Paolo Alatri, in una rassegna di «Le riviste di cultura» dell'epoca (Rinascita, VII, 1, gennaio 1950, p. 32-37), affermava che «in campo cattolico, si può dire che il silenzio regni sovrano. Alla eccezionale predominanza politi$\mathrm{ca}$, che i cattolici sono riusciti a conquistarsi in Italia negli ultimi anni, non corrisponde neppure di lontano una degna posizione culturale che possa esprimersi attraverso la stampa periodica. Si ripete qui, in sostanza, quel fenomeno che Pietro Ingrao metteva giustamente in rilievo a proposito della stampa quotidiana cattolica [Rinascita, ottobre 1949, p. 436]: «il peso dell'influenza clericale sull'opinione pubblica non si esprime attraverso adeguati strumenti di egemonia culturale, che i cattolici non detengono affatto». 
classe operaia a sua volta nazionalmente vocata, come Gramsci l'aveva proposta e Togliatti tentava di realizzare nella prassi politica.

Al lavoro degli storici comunisti veniva insomma affidato il compito di supportare un'operazione politica di trasformazione del nazionalismo dei ceti medi, cui era approdata l'idea di Patria formatasi nel Risorgimento, in un nuovo patriottismo compatibile con una nozione di internazionalismo proletario affatto diversa, anzi aliena dal cosmopolitismo grande borghese; un patriottismo riconducibile se mai all'ideologia 'patriottica' che era stata del giacobinismo, nell'accezione s'intende di quel realismo politico alieno dall'astrattezza settaria con cui Gramsci lo aveva nazionalmente opposto alla strategia di 'termidorismo preventivo' poi adottata dai moderati del Risorgimento, Cavour in primo luogo. Si dispiegò, su questo punto decisivo, una campagna sistematica, articolata nei saggi e recensioni dei periodici e in articoli di quotidiano. ${ }^{7}$ Sottolineo la particolare importanza del richiamo a questo 'patriottismo giacobino' (nel senso gramsciano equivalente a 'nazional-popolare') come criterio storiografico teso a rovesciare sia la tradizione 'nazionalistica' della borghesia imperialista sia la 'municipalistica' del sanfedismo, e insieme a contrastare l'insidiosa tendenza alla disidentificazione nazionale comportata dalla nuova ideologia dell'americanismo globale. Anche su questo punto, pure assai interessante, un solo esempio a titolo esemplificativo, la risposta di Renato Zangheri alla rivista Il Mulino, che aveva attaccato la politicizzazione della storiografia sovietica e messo in discussione appunto l'uso storiografico dei criteri di patriottismo e di cosmopolitismo.

Zangheri si chiedeva polemicamente «se nazioni, movimenti nazionali, apporto di ogni nazione alla cultura mondiale, contino qualcosa nella storia, e siano individuabili e definibili. Vi è chi lo asserisce e fra questi i marxisti; i quali tuttavia si differenziano dai ricercatori del colore locale, dai mistici dello spirito nazionale, e simili. V'era anche chi lo negava. Ed erano spesso nobili spiriti, mossi da un sogno di universale concordia. Oggi però il cosmopolitismo, nella sua essenza, è diventato altra cosa. Si nega e deprime il patrimonio nazionale altrui per esaltare il proprio, e neanche la sua parte migliore. Si invitano le nazioni d'Europa a dimenticare il passato che le fece grandi, e si afferma la supremazia americana. Per questa via si arriva a formulare una direttiva storiografica, e si falsifica la storia dei popoli». La questione aveva valenza primaria, ed è significativo che in Rinascita, a un articolo intorno al capitale tema desanctisiano 'la scienza e la vita', ne facesse seguito un altro su "Lotta nazionale e lotta di classe nel Viet Nam», in cui —oltre al tema nazional-popolare della rivoluzione 'patriottica' nel senso giacobino di

7. Cito per tutti, in questa sede non posso fare di più, il saggio «Dal nazionalismo al patriottismo" (Rinascita, VII, 11-12, novembre-dicembre 1950, p. 529-32) di Lucio LOMBARDO RADICE: lo scelgo per la particolare personalità dell'autore, matematico di vaglia, un altro marxista e comunista proveniente da famiglia di grande tradizione idealistica (era figlio del pedagogista Giorgio), un interprete fra i più intelligenti della linea politico-culturale di Togliatti. 
nazionale e sociale insieme - veniva messa in rilievo la natura ormai affatto americana dell'impegno francese in Indocina. Quanto a criteri interpretativi disidentificanti di origine statunitense che riguardavano da vicino l'Italia, un caso tipico era quello, per esempio, della questione meridionale costretta nella categoria 'mondializzante' delle aree sottosviluppate. ${ }^{8}$

\section{Battaglia politica, conflitto storiografico}

Il Risorgimento, politiche economiche e linee culturali, divenne dunque — soprattutto dopo l'uscita di Risorgimento e di Materialismo storico e la filosofia di Benedetto Croce del Gramsci e nelle forme della disputa storiograficaterreno di una contesa ideologica particolarmente acuta: De Sanctis ne fu al centro, e d'altronde non si può dimenticare che il mito (o, se si vuole meglio, la crucialità) risorgimentale di De Sanctis era stato a sua volta costruito dal Croce come uno dei cardini essenziali - Vico, la sconfitta giacobina del 1799, Cuoco, i Poerio-Imbriani-Spaventa, De Sanctis, poi Giolitti- della sua 'nuova Italia', e che proprio su questa base intrinsecamente 'politica' De Sanctis era diventato - come Vico in filosofia - il suo precursore nazionale in estetica e critica letteraria. Certo nella Storia d'Italia del 1927 De Sanctis non era più il rappresentante non dottrinale del materialismo storico come Croce l'aveva definito [1896] quando il suo filosofo italiano di riferimento era ancora il marxista Antonio Labriola, ma uno dei crociani più ferventi e combattivi, il già rammentato Luigi Russo, aveva subito fatto seguire nel 1928 l'inquieto libro su De Sanctis: e proprio ad esso Gramsci si era specialmente riferito per il suo ritorno a De Sanctis in opposizione al ritorno fascista proposto da Gentile. Perché, mentre Gramsci in carcere ragionava sul libro di Russo (su quel che poteva intuirne, conoscendolo solo attraverso recensioni), Gentile - contro la linea desanctisiana Croce-Russo - si stava appunto preoccupando di rivendicare, emblematicamente sul numero inaugurale dell'ambiziosa rivista fascista $Q u a-$ drivio, un De Sanctis - con gli Spaventa - precursore del fascismo e della sua ideologia della cultura come azione. ${ }^{9}$ Non si può dimenticare, voglio dire, che De Sanctis e la sua fortuna erano sempre stati legati ad un'interpretazione politica del recente Stato unitario, della formazione sua e dei suoi gruppi dirigenti, delle sue prospettive: una fortuna insomma radicata - fin dalla pionieristica rivendicazione crociana- in ragioni storico-politiche piuttosto, o comunque prima che storico-letterarie.

8. Per un cenno al dibattito su questo punto e a come si intrecciasse con la discussione etnologica e letteraria rinvio a U. CARPI, «Scotellaro 1954, l'intellettuale conteso», Nuova Rivista di Letteratura Italiana, VII (2004), 1-2, p. 383-410.

9. Il Quaderno 23 del 1934 si apriva per l'appunto con un essenziale paragrafo (significativamente già impostato nel Quaderno 17 all' interno di una riflessione sulla 'letteratura popolare') «Ritorno a De Sanctis», sollecitato dal recente «Torniamo a De Sanctis!» di Gentile (Quadrivio, I, 1, 6 agosto 1933, p. 3). Sarà interessante sapere che l'articolo di De Sanctis era incastonato fra due liriche rispettivamente di Ungaretti, 1914-1915 e di Cardarelli, Sardegna. 
Non deve dunque stupire che l'ultimo Croce, con i suoi fedelissimi di «Nord e Sud» del «Mondo» dello «Spettatore Italiano», venisse combattuto in modo così aspro dagli intellettuali comunisti — dei quali non a caso era intanto diventato autorevole 'compagno di strada' quel Russo desanctisianamente suggestivo per il Gramsci dei quaderni- come colpevole di aver negli anni, sostanzialmente a partire dall'Estetica, progressivamente abbandonato, anzi rovesciato, le posizioni innovatrici della giovinezza (diciamo la linea labrioliano-desanctisiana) approdando a posizioni conservatrici e fin reazionarie. ${ }^{10}$ Né deve stupire che nel medesimo tempo quegli stessi intellettuali, nelle loro 'battaglie delle idee' combattute contro i liberali sui temi della libertà e partiticità della cultura, non esitassero a richiamarsi proprio — sempre naturalmente attraverso, esplicita o sottintesa, la mediazione gramsciana- alla tradizione borghese e progressiva della linea De Sanctis-Croce ${ }^{11}$, fondandovi le ragioni della nuova prospettiva De Sanctis-Gramsci. Nell'infinita casistica, vale la pena di citare ancora, per la sua lucidità, Zangheri:

La scienza e la vita. Non vi farò il torto di ripetervi il già noto. Non vi citerò il De Sanctis. Ma bisogna ammettere che anche nello specifico campo della produzione storiografica ha avuto luogo quella corrispondenza. Corrispondenza che è veramente una unità, la «unità del pensiero storico con l'attualità della vita»: come scrisse il maestro che ora ci ha lasciati. È vero che il Croce quella unità non volle poi tenerla ferma e la annullò, volgendola in speculazione. Ma allorché voi inorridite di questa funzione pratica e di strumento insomma della società che vi pare sia attribuita alla storiografia sovietica, vorrei ancora richiamarvi il pensiero crociano che "tutta la scienza, tutta la cultura storica, specificamente elaborata e promossa, sta in rapporto al bisogno generale di matenere ed accrescere la vita civile ed attiva dell'umana società».

Il dilemma fra resistenza di De Sanctis-Croce ovvero emergenza di De Sanctis-Gramsci alludeva insomma a ben altro che ad una specialistica questione di metodologia storiografico-letteraria, era — tanto più se posto nei termini dell'eredità storica piuttosto che della mera contrapposizione- la metafora di una battaglia culturale per la conquista della nuova egemonia politica. Ed è da notare che questo insidioso riallacciarsi dell'intellettuale marxista a De Sanctis e a Croce in funzione della militante politica comunista, insomma l'atteggiarsi dei comunisti ad eredi per conto della classe operaia del liberalismo progressivo, irritava particolarmente quelli del «Mulino»: «tu presenti il Partito Comunista sulla linea di sviluppo del liberalismo, e citi De Sanctis e Croce. Se è vero che questi nostri pensatori già stabilirono un nesso tra politica e storiografia [...] questo nesso non fu per loro quello che è per te o, più precisamente, per

10. Memorabili le polemiche col Croce combattute da Togliatti alias Roderigo di Castiglia, e ricordo fra tutte la celebre nota "Monotonia e vacuità dell'anticomunismo crociano", Rinascita, IV, 8-9, agosto-settembre 1949, p. 357-59 in risposta al «Monotonia e vacuità della storiografia comunista» del Croce.

11. Dalle già citate pagine del gennaio 1953 in polemica col Mulino sulla politicità della cultura, nel caso la politicità-partiticità del lavoro storiografico. 
Lenin e Stalin. (Questo di prendervi a tutti i costi dei protettori o precursori gloriosi e autorevoli, è un malcostume culturale che non può esservi facilmente perdonato...)».

Battaglia culturale? L'evocazione di una terminologia bellicista non sembri fuori luogo o eccessiva: all'aggressività della politica culturale comunista -mentre lo schieramento cattolico rispondeva soprattutto con l'azione capillare delle organizzazioni di massa (Azione Cattolica, universitari della F.U.C.I., Coltivatori diretti ecc.) — il mondo laico, la cosiddetta 'terza forza', rispondeva con una vera e propria controffensiva antimarxista. Il più incisivo dei periodici liberal-repubblicani (Il Mondo settimanale), annunciava l'uscita a Napoli della rivista "Nord e Sud», nata in ambiente crociano col precipuo scopo di contrastare l'offensiva della cultura marxista nel Mezzogiorno e sulle cui pagine di lì a poco Rosario Romeo avrebbe sferrato un attacco frontale contro l'interpretazione gramsciana del Risorgimento, con una nota dal titolo inequivocabile «Fronte del Sud». ${ }^{12}$ La quale nota si concludeva, alla stregua di un bollettino bellico, con l'annuncio che "gli amici di Nord e Sud conducono, come ci auguravamo ed avevamo previsto, una 'buona guerra'» finalizzata ad «aver ragione di un avversario rotto a tutte le astuzie», di cui la più insidiosa era proprio questa capacità di «farsi passare per gli eredi autentici del pensiero di un De Sanctis, di uno Spaventa o di un Labriola».

La stampa comunista, che nell'ambito di una grande campagna meridionalista era per prima passata all'attacco nel 1954 lanciando a Napoli le mensili Cronache meridionali con immediata preoccupazione dello schieramento laico, reagì all'uscita di Nord e Sud opponendo sul Contemporaneo un impegnativo articolo, notevole anche a posteriori né solo per la firma di Giorgio Napolitano, dal titolo che, con l'integrazione del sottotitolo riassuntivo ( Nord $e$ Sud riconosce ai comunisti il merito di aver promosso il moto di liberazione delle plebi meridionali, ma insiste a indicare nel comunismo il pericolo numero uno del Mezzogiorno d'Italia»), era tutto un programma, «L'acqua nel mortaio». ${ }^{13}$ La neonata rivista napoletana controrispose con una nota redazionale, «Le stoccate di Ferravilla», in cui ribatteva essere il disegno comunista nientemeno che quello di «catturare le energie politiche del Mezzogiorno per poterle rivolgere contro le istituzioni dello Stato democratico». Di questo disegno, era sottinteso, Vico De Sanctis Labriola Croce rappresentavano la strumentale copertura culturale, ed ecco perché — con Napoli essendo in gioco l'Europa, l'Occidentetanto accanimento da ultima barricata nella contesa storico-filologica sui padri. $^{14}$

12. Il 29 marzo 1955.

13. G. Napolitano, «L'acqua nel mortaio», Il Contemporaneo, II, 1, 1 gennaio 1955, p. 1-2. Per la campagna meridionale lanciata dai comunisti nel 1954 ricordo, fra gli innumerevoli, due interventi particolarmente importanti di impostazione politica generale (P. TOGLIATTI, "L'azione democratica e socialista nel Mezzogiorno», Cronache meridionali, I, 6, giugno 1954, p. 401-22) e di prospettiva politico-culturale (M. ALICATA, "Il meridionalismo non si può fermare a Eboli», Cronache meridionali, I, 9, settembre 1954, p. 604-16).

14. Nord e Sud, II, 3, febbraio 1955, p. 32-35. 
Riferimento culturale Croce, riferimento politico dei napoletani di «Nord e Sud» era invece Giorgio La Malfa, il vero, intelligente interlocutore meridionale di Amendola, Napolitano, Alicata: sul primo numero della rivista pubblicò un saggio, «Mezzogiorno nell'Occidente», nel quale sviluppava in prospettiva economico-politica attuale la tesi crociana del respiro europeo della cultura e degli intellettuali meridionali. Osservo che anche La Malfa proveniva da quella Banca Commerciale, dove era in allestimento la collezione ricciardiana dei classici sopra rammentata. Tutto un mondo borghese di cultura laicoliberale democratica e illuminista (questo singolare asse filosofico-bancario fra Milano e Napoli attrasse e mise in movimento, come ho accennato, anche la Torino gobettiana, si pensi a cosa è stato in quella collezione e quale patrimonio continua a rappresentare per noi il Settecento ordinato per la Ricciardi da Franco Venturi e da Mario Fubini) che è scomparso lasciando un vuoto incolmato, e sul quale si capisce come i più intelligenti dei comunisti, i Togliatti gli Amendola i Napolitano gli Alicata, potessero pensar di innestare la nuova linfa riformatrice e democratica del movimento operaio. Il pregiudizio anticomunista da un lato, i condizionamenti internazionali dall'altro (se li rinfacciavano a vicenda questi limiti, e avevano entrambi ragione e insieme torto, assicurando così — con questo loro reciprocamente paralizzante conflitto — una vera e propria rendita di posizione per la pragmatica Democrazia Cristiana ...) impedirono una sintesi che forse avrebbe consentito all'Italia intera, alla stessa Democrazia Cristiana e al Partito Socialista, una diversa evoluzione. Ma questo è un altro discorso.

Quel linguaggio (quella logica) conflittuale era evidentemente ineluttabile. L'Unità del 20 gennaio 1955, per esempio, pubblicò un articolo del filosofo più prestigioso nel Partito, il Cesare Luporini ben noto anche agli italianisti per l'innovativo saggio del 1947 Leopardi progressivo in cui aveva rovesciato il giudizio crociano sulla 'vita strozzata' e insieme valorizzato la critica mossa da Leopardi al moderatismo e spiritualismo dei gruppi dirigenti del Risorgimento ${ }^{15}$, e quell'articolo di Luporini il quotidiano del PCI lo intitolò appunto «Il fronte filosofico». Non meravigli che un quotidiano pubblicasse articoli di questo genere, anche a destra accadeva che per esempio il Giornale d'Italia

15. Il Luporini all'inizio del 1954 aveva pubblicato un libro su La mente di Leonardo - compiendo per il rinascimentale Leonardo un'operazione analoga a quella compiuta per il risorgimentale Leopardi, di rivalutazione cioè in senso progressivo della mente contro il giudizio cociano di a- ovvero anti-filosoficità- molto importante anche per una definizione ed un uso storicamente determinati di quel concetto di realismo che era poi il vero nocciolo teorico-politico della concomitante querelle desanctisiana (da leggere è anche la recensione di L. LOMBARDO RADICE, «Un libro di Cesare Luporini: La mente di Leonardo», L'Unità, 5 febbraio 1954, in cui venivano puntualmente richiamate, in uno con la polemica antimedievalista e antispiritualista, le problematiche rinascimentali di De Sanctis e di Gramsci). Ricordo che Luporini, il quale aveva alle spalle una formazione assai complessa essendo stato a suo tempo allievo anche di Heidegger, fu in quel periodo il protagonista forse di maggior spessore e prestigio delle discussioni filosofiche — combattute anche sul fronte interno, basti pensare a quelle con la scuola di Della Volpe — intorno alle prospettive del materialismo dialettico. 
entrasse in filosofia stampando, come aveva fatto il 13 marzo 1949, un grande Dialogo immaginario di Croce con Hegel, nel quale il vecchio filosofo si era preoccupato di espungere dalla storia del proprio incontro con Hegel quel che aveva riconosciuto fino al 1915, cioè la sostanziale mediazione, per arrivare a quell'incontro, del marxista Labriola e del marxismo storico. Ricordo il fatto perché, per comprendere quanto il terreno —o se si vuole il fronte- filosofico (ovvero estetico-letterario) fosse sentito ravvicinatamente, anzi intrinsecamente e quasi immediatamente politico e politico-formativo, mi sembra significativo che gli stessi quotidiani e rotocalchi ritenessero di darne informazione ai propri lettori. ${ }^{16}$

Fronte molto ampio il filosofico prospettato da Luporini ai lettori dell' «Unità» (par di sognare, con quel che passa oggi il convento del giornalismo politico italiano!), fronte che andava dalle più aggiornate forme di neospiritualismo e irrazionalismo fino all'empiriocriticistico neopositivismo logico contro cui veniva invocato l'allora canonico Lenin di Materialismo ed empiriocriticismo (ma non farebbe poi troppo male rileggerlo anche oggi...). In quel fronte (espressione ereditata dal lessico politico leniniano) di guerra filosofica la trincea di base si confermava peraltro l'apologia in chiave labrioliana e gramsciana delle «più vigorose fra le correnti ideali che avevano accompagnato la formazione dello Stato borghese in Italia e che si esprimevano nei nomi di Spaventa e De Sanctis» per rovesciare il tentativo compiuto dall'idealismo crociano e gentiliano di unificare e dirigere la cultura italiana in nome della borghesia. E il fatto che questo tema venisse ripreso in un articolo di orientamento politico proprio da un filosofo come Luporini, fra gli intellettuali comunisti italiani il meno toccato dalla tradizione idealistica nazionale (insieme con l'Antonio Banfi già allievo di Simmel e di Husserl e col materialista antistoricista Ludovico Geymonat, mentre diverso - sempre con qualcosa di costitutivamente 'esterno'- fu il caso di Della Volpe e della sua scuola), conferma ulteriormente che si trattava di una linea d'intervento sistematica e forte, senza nulla dell'estemporaneo e gratuito che aduggia le deboli schermaglie odierne.

Sistematica e forte, altrettanto sistematicamente e fortemente contrastata proprio su questo punto della tradizione nazionale, che era un autentico nervo scoperto. I comunisti celebravano l'ottantesimo compleanno di Thomas Mann ${ }^{17}$ ? "Il Mondo" replicava tornando a battere sul dente che più doleva: «Ma tant'è, i comunisti si ostinano a celebrare proprio i campioni della civiltà capitalistica, a preferire al realismo socialista quello borghese, ad inneggiare a Dreiser e a Mann, o, per restare in Italia, a Verga e De Sanctis e Spaventa

16. Il veleno politico del dialogo immaginario venne puntualmente rilevato da un giovane studioso marxista di filosofia, Giovanni Mastroianni, che ritroveremo fra poco a puntualizzare filologicamente e bibliograficamente nelle polemiche vichiane. Per l'occasione inviò una lettera a Rinascita, 5 maggio 1949, p. 240, dalla redazione titolata «La discendenza HegelCroce».

17. Una celebrazione effettivamente vistosissima: bisognerebbe rivedere le ragioni del giudizio su Mann espresso dalla cultura marxista italiana, né solo comunista. 
e Labriola, e, per un po' che i loro interlocutori si distraggano, anche Croce». ${ }^{18}$ L'obiezione di fondo, la medesima dal Mulino al Mondo, era ingenua: perché i nostri comunisti hanno bisogno di cercare un avallo nei campioni del pensiero borghese e non si avvalgono (o non si limitano ad avvalersi) di quelli del loro Pantheon marxista? Era proprio questa separatezza, questa concezione del conflitto delle classi come una sorta di incomponibile scissione della storia e della società e dei valori che lo storicismo dialettico di Gramsci e di Togliatti rifiutava: dal proletariato antinazionalista e dalla separazione-incontaminazione culturale delle classi subalterne al popolo nazionale e alla cultura nazional-popolare, dalla scissione rivoluzionaria allo storicismo progressista, questo marxismo 'desanctisiano-labrioliano' spostava i termini consueti della discussione. Li spostava al punto di aprire acuti conflitti, oltre che nel fronte 'liberale' (Bobbio e Nuovi Argomenti mostravano tutt'altra apertura e interazione che Mondo e Nord e Sud), anche sullo stesso fronte interno, a sinistra, con sempre più diffuse manifestazioni di insofferenza, destinate ad esplodere negli anni Sessanta, per questo che - se ai crociani pareva un' usurpazione- a molti marxisti cominciava invece a sembrare un pericoloso scivolamento sul terreno dello storicismo idealistico: né solo ai marxisti 'critici' di quei settori che evolveranno ad extra-sinistra, ma anche ai più ortodossi marxisti-leninisti (Sereni, Aloisi), impegnati infatti in un'interpretazione di Gramsci condotta secondo canoni più rigidamente, come si diceva, marxisti-leninisti. ${ }^{19}$

Guerra ideologica per guerra ideologica, in quei mesi fra 1954 e 1955 i punti di fuoco erano molti, i colpi talvolta sviavano da un campo all'altro con non pochi episodi di quel che oggi si chiama rischio da 'fuoco amico', gli strateghi faticavano a tener sotto controllo il senso generale della battaglia: fuor di metafora, una straordinaria stagione di confronto politico-culturale, come ad una svolta decisiva per la conquista dell'egemonia. Ogni occasione di dibattito anche stagionale per un film o per un romanzo, ovvero per questioni di più lunga durata dal nesso libertà-uguaglianza fino all'uso industriale dell'energia nucleare e in genere della scienza, ovvero ancora per le prospettive di organizzazione e indirizzo della ricerca storica o filosofica, tutto diventava motivo di serrato confronto politico. E tutto stava dentro o veniva comunque ricondotto sempre alla questione essenziale dell'identità e unità nazionale, all'interpretazione del suo formarsi e delle sue forze motrici dal Risorgimento alla Resistenza e alla Repubblica. Politicità e partiticità della cultura: non solo per disputare o disputarsi la storicità di De Sanctis o Gramsci, ma la contingenza dell'operaio Metello, dei cafoni di Tricarico, dei giorni d'amore del regista

18. «L’omaggio comunista», Il Mondo, 23 agosto 1955, p. 2.

19. Sereni già nel 1948 («Gramsci e la scienza di avanguardia», Società, IV, 1, gennaio-febbraio 1948, p. 3-30); Aloisi nel 1950 ("Gramsci, la scienza e la natura come storia», ibid., VI, 3, settembre 1950, p. 385-410: dall'intervento di Aloisi nacque un'interessante discussione con il Matteucci filosofo di punta del Mulino (N. MatteuCCI-M. AloisI, "Gramsci e la conoscenza della natura», Emilia, dicembre 1950, p. 400-04, e analoghe discussioni successive non ebbero più la medesima vivacità e freschezza politica) sul dilemma —oggi affatto storico-filosofico, allora tutto politico- Gramsci-Croce ovvero Gramsci-Marx/Lenin. 
De Santis, del primato sui dieci anni dalla fine della guerra ovvero le prospettive 'materialiste' della biologia (scienza assai più sospetta di deviazionismi idealistici era considerata la fisica...) e i cieli della declinazione politica della libertà, dei destini dell'arte, del rapporto globale fra Occidente e Oriente.

Chi ne abbia memoria, si sarà accorto che ho puntualmente alluso ad alcune allora clamorose e fra loro intrecciantisi contese culturali di quegli anni, particolarmente concentrate fra il 1954 e il nostro 1955, alle soglie del $1956 ;{ }^{20}$ protagonisti, spesso, i medesimi politici e intellettuali: sempre, le medesime ragioni politiche. Con un nesso strettissimo fra queste ragioni e la ricerca storica, particolarmente rilevabile proprio nella nostra questione desanctisiana: che si articolava infatti, autentica strategia storiografica, in varie direzioni intrinsecamente connesse, da Vico agli Spaventa a Labriola, le tessere da rovesciare della linea crociana. ${ }^{21} \mathrm{Ma}$ tutto ciò accadeva (dimenticarlo significherebbe perdere il senso complessivo di una stagione in cui i diversi piani di movimento, economico-produttivo, ideologico, politico, artistico, ognuno sul proprio terreno pullulante di vitalità e di contraddizioni, si scivolavano spesso

20. Le querelles su Metello e su Scotellaro sono ben note. La discussione sulla libertà è pure molto conosciuta e coinvolse, fra Nuovi Argomenti e Rinascita, soprattutto Bobbio e Togliatti. Il dibattito sulla biologia (nel primo decennio postbellico fu ampia la discussione sul rapporto fra ricerca scientifica e materialismo, altra tessera delicatissima delle relazioni con la cultura idealistica) fu guidato particolarmente su Società dall'Aloisi; dell'arte e di estetica si discusse per ogni dove, ma (insieme alla discussione del Contemporaneo, dicembre 1954, su marxismo, arte ed estetica) soprattutto va ricordata una grande inchiesta di Nuovi Argomenti e sulla medesima rivista anche la discussione su Oriente e Occidente. Dieci anni si intitolò una lunghissima inchiesta del Contemporaneo 1955, peraltro ripresa da varie altre testate, sul decennio intercorso: al fondo, l'interrogativo angoscioso e politicamente stringente se la Resistenza fosse stata — anche dal punto di vista culturale - tradita. La discussione meno nota e apparentemente più datata, ma per noi molto significativa perché tutta incentrata, come quella per Metello, sulla questione del realismo, riguardò il film di De Santis Giorni d'amore (pellicola oggi di arduo reperimento ancorché all'epoca di gran successo, protagonisti, per la cronaca, Marcello Mastroianni e Marine Vlady) e si svolse sull'edizione settentrionale dell' Unità nel nostro 1955. Come si vede, il campo di battaglia desanctisiano era illuminato anche dai fuochi di altri e contigui terreni di scontro (ma una vivacissima discussione su cinema e neorealismo, nell'estate, si svolse pure sull'Avanti.).

21. Questi nessi politici non sfuggirono a un’ intelligente rassegna di Giuseppe VACCA («Recenti studi sull'hegelismo napoletano", Studi Storici, VII, 1, gennaio-marzo 1966, p. 159-209), il quale mise puntualmente in luce come a distanza di dieci anni (altri dieci anni, ma un lasso ormai da calcolare per progressione geometrica, non sommatoria...) quella stagione togliattiana fosse diventata delicato oggetto di serrato confronto politico: che si aprì per esempio fra Rossana Rossanda («Le ragioni della cultura. Note e appunti critici su 'impegno, cultura e ideologia'», Il Contemporaneo, febbraio 1965, p. 1-4) e Luciano GruPPI ("Palmiro Togliatti, cultura e metodo», Rinascita, 18 settembre 1965, p. 23-24, cui Rossanda replicò con "Marxismo e storicismo", ibidem, 13 novembre 1965, p. 22-23) emblematicamente a un anno dalla morte di Togliatti e già quasi in termini di resa dei conti (proprio a partire da un numero commemorativo del Contemporaneo, nel frattempo trasformatosi in supplemento mensile di Rinascita). Argomento delicato ancor adesso pur in un secolo affatto diverso, peraltro divenuto oggetto di riflessione storica e non più, come allora, di confronto militante: capire le origini del declino che ci ha condotto ad oggi, l'astrattezza — politica senza responsabilità — di Rossanda, il ripetitivo buon senso —occhi all'indietro— di Gruppi. 
accanto senza l'uno incrociar l'altro ovvero talvolta collidendo) in un tempo inquieto: discutevano dell'esemplarità operaia di Metello e dell'autonomia culturale dei contadini del Sud, e intanto a Roma Pasolini e Patroni Griffi facevano scappar fuori l'emarginazione dei ragazzi di vita ${ }^{22}$ e a Milano Pagliarani cominciava a dar forma alle nevrosi metropolitane della ragazza Carla; era sotto i torchi il primo volume della Storia dell'Italia moderna di Candeloro (una lunga serie che sarebbe giunta fino agli anni Ottanta in leale fedeltà alla linea interpretativa De Sanctis- Gramsci) mentre di Gramsci i versi pasoliniani invitavano a interrogare ormai le ceneri.

Il più ampio dibattito nazionale del 1956, a parte quello promosso da Nuovi argomenti con le famose «10 domande» su Stalin e stalinismo, si svolgerà non a caso, come per riprendere le fila e riaccordare i piani, sul tema La cultura marxista: il primo intervento, senza De Sanctis e senza Labriola, Stato d'assedio.

\section{Sul fronte idealistico, dal liberalismo al marxismo: per Spaventa e per Labriola}

De Sanctis? Ma in discussione era tutto l'hegelismo napoletano, a partire da Croce (ovvero per giungere all'antiCroce). Hegel e Croce, intervenne Mario Alicata, ${ }^{23}$ in quel momento vero regista operativo - soprattutto in questioni meridionali- della politica culturale comunista: e fu uno dei casi in cui i proiettili fischiarono anche fra le linee amiche. Il fatto è che Lombardo Radice, stroncando sulla classica linea leniniana della critica all'empiriocriticismo i Lineamenti di una teoria dei segni di Charles Morris tradotti e proposti alle scuole italiane da Ferruccio Rossi-Landi, aveva concluso esser comunque preferibile, a questo scientismo neoempirista, l'Estetica di Croce e, per restar fedeli alla dialettica, meglio esser sentenziati «marxisti imbevuti di idealismo». ${ }^{24}$ Alicata, pur convenendo che queste nuove correnti di pensiero erano estranee alla linea tradizionale di sviluppo del pensiero italiano e anche in quanto tali da combattere, respingeva qualunque contestuale intiepidimento della battaglia contro l'idealismo hegeliano nei modi che ha assunto in Italia con Benedetto Croce. Anzi, proprio questo della linea tradizionale doveva restare il fronte principale,

22. Di questi due libri fece una stroncatura politica Giovanni BERLINGUER ( $I l$ vero e il falso delle borgate di Roma», l'Unità, 29 luglio 1955), indisponibile a leggere in quei termini il popolo romano delle borgate.

23. M. AliCaTA, «Hegel e Croce», Il Contemporaneo, II, 33, 20 agosto 1955, p. 4.

24. L. Lombardo RADiCE, "In principio era il Segno», Il Contemporaneo, II, 32, 13 agosto 1955, p. 11: «Dite pure: marxisti imbevuti di idealismo. Non è un insulto. Anzi, è una verità storica quando si dica: marxisti imbevuti di quella dialettica della quale ebbero una prima consapevolezza i grandi filosofi dell'idealismo». La polemica continuò, con replica di Lombardo Radice e conclusione dello stesso Alicata, coinvolgendo anche Badaloni, Della Volpe, Colletti. Andrebbe riletta, soprattutto come sintomo dell'insoddisfazione ormai latente per l'arroccamento nella vecchia formula antiempiriocriticista a fronte del nuovo antistoricismo neopositivista e neoempirista. 
e proprio per la sua specificità nazionale: l'egemonia culturale, in Italia e nell'ottica ancora fortemente postrisorgimentale del Risorgimento senza rivoluzione e della Resistenza come secondo Risorgimento, la si conquistava certo anche con il contrasto a Morris sul terreno della circolazione mondiale delle culture, ma prima di tutto sconfiggendo lo storicismo idealistico come Croce lo aveva nazionalmente declinato e incardinato. Che era una prospettiva di lotta filosofica sostanzialmente analoga al fronte prospettato in gennaio da Luporini.

Sconfiggendolo, ripeto, nella lettura crociana, ma facendone proprie le originarie ragioni di rivoluzione nazionale, riprendendone il filo progressivo che lo stesso giovane Croce nei suoi anni labrioliani aveva tenuto in mano e poi abbandonato, anzi tessuto per una diversa tela antisocialista e regressiva. Uno storico marxista di appartenenza socialista ma fortemente e stabilmente impressionato da Gramsci, Gaetano Arfé, già nel 1952 aveva affrontato il problema, prendendolo per il corno più difficile, proprio quel Bertrando Spaventa nel cui Stato etico il Gentile aveva fissato l'origine risorgimentale dello Stato forte fascista, mentre nella sua Destra hegeliana (e di De Sanctis nella Sinistra) Croce aveva cercato il fondamento dell'Italia liberale. Sulla scia di Gramsci, diceva l'Arfé, ${ }^{25}$ molto si sta facendo per De Sanctis, ma occorre ritornare anche a Spaventa, riprenderne, contro quelle di Gentile e di Croce, l'interpretazione di sinistra datane da Labriola: e puntare sull'hegelismo, filosofia rivoluzionaria nel contesto italiano dominato dal neoguelfismo di Gioberti e dal clericalismo dei gesuiti. Né solo Hegel, ma anche il Giordano Bruno dello Spaventa, la tradizione italiana dei pensatori ribelli del Rinascimento, Bruno Vanini Campanella Vico: sintonia, notava Arfé, fra la storia filosofica nazionale di Spaventa e la letteraria di De Sanctis, mentre Croce ha depurato la tradizione idealistica di ogni punta eretica, di ogni data rivoluzionaria. «In realtà — secondo ArféBertrando Spaventa è tra quei pochi uomini del Risorgimento che sentano il liberalismo come fatto rivoluzionario, e postulino come necessario il momento della rottura, del distacco netto dal passato»: e dunque, se è una minoranza quella che si è assunta il compito di dirigere il movimento nazionale, essa deve avere la forza e il coraggio di «assegnare allo Stato, perciò concepito come potenza etica, il compito preciso di creare e attuare nuove forme di socialità e di vita morale». Certo vi era implicita la possibilità di una reazionaria interpretazione statolatrica alla Gentile (illiberale per Croce, antidemocratica per Gramsci); ma una lettura dal punto di vista gramsciano consentiva ad Arfé, e c'era in nuce il programma della riforma morale e culturale, di rovesciare il ragionamento come problema «di ottenere l'adesione spontanea, il consenso dei cittadini allo Stato sulla base di un'egemonia esercitata attraverso il diffondersi di una ideologia nuova laica e liberale». Un modo hegeliano-dialettico di pensare, che prepara il terreno - Labriola iniziatore- all'accoglimento del marxismo.

25. G. ARFÉ, «L'hegelismo napoletano e Bertrando Spaventa», Società, VIII, 1 marzo 1952, p. $45-62$. 
Mi sono soffermato sull'articolo di Arfé perché nel modo più netto, se vogliamo più schematico, vi sono delineate ragioni ed obiettivi della politica culturale storicistico-marxista e nazionale-marxista di quegli anni, il programma di lavoro per De Sanctis e per Labriola: Spaventa, la rottura hegelianoliberale della borghesia laica e poi De Sanctis, il realismo, il modello del borghese progressivo; ma cruciale, per il nesso con la via gramsciana e per il passaggio dall'egemonia della classe borghese all'egemonia delle classi lavoratrici, Labriola, storicisticamente l'anello marxista sul terreno della cultura. Una battaglia anticrociana a tutto campo.

Più si approfondisce la storia di Antonio Labriola, più ci si sforza, cioè, di intenderne l'opera.

Situandola nel posto che le spetta rispetto alle sue premesse e alle sue conseguenze, più si rivela.

Inadeguata la pretesa, durata a lungo sulle orme del Croce, di considerarlo una figura geniale ma isolata nella storia della cultura italiana. Da Francesco De Sanctis, da Bertrando Spaventa fino ad Antonio Gramsci ci appare, oggi, il disegno dello svolgimento ideale di uno storicismo italiano diverso da quello crociano, e che passa attraverso Labriola, anzi fa cardine nel suo pensiero. Corrente italiana, non nel senso angusto e meschino che fustigava Gramsci, ma nel senso più vero, perché fecondata nel continuo connubio col pensiero europeo da Hegel a Marx a Lenin. ${ }^{26}$

Uno storicismo italiano diverso da quello crociano, da De Sanctis e Spaventa al Gramsci ordinovista per la via di Labriola, ecco il disegno, col Partito Comunista che si propone erede, dopo il secondo Risorgimento, delle forze trainanti risorgimentali: e con un marxismo italiano, per queste sue radici, intrinsecamente diverso dal 'positivistico' delle socialdemocrazie europee di matrice secondointernazionalista. Vero? Non vero? Il problema era prima di tutto politico, se cioè fosse vera l'ipotesi strategica a cui questa linea culturale voleva esser funziona-

26. Da un corsivo redazionale "Per il cinquantenario di Antonio Labriola», Società, X, 3, maggio-giugno 1954, p. 402-05, cui seguiva, p. 406-30, G. BERTI, «Bertrando Spaventa, Antonio Labriola e l'hegelismo napoletano" (econcluso in due puntate successive, nel luglioagosto, p. 583-607 e nel settembre-ottobre, p. 764-91). La proliferazione di interventi sugli Spaventa e su Labriola nella stampa comunista fu notevole. Qualche indicazione: M. SPINELLA, "Per un ritorno ad Antonio Labriola», Emilia, gennaio 1954, p. 9-11; L. CAFAGNA, «La critica di Labriola», Il Contemporaneo, II, 2, 8 gennaio 1955, p. 3 (diverso dagli altri, questo intervento non parte dalla collocazione del Labriola rispetto all'hegelismo, bensì ne esamina la polemica contro le tendenze opportunistiche e la debolezza teorica del riformismo di Turati); M. AliCATA, «Ricordo di Antonio Labriola», L'Unità, 2 febbraio 1955; D. CANTIMORI, «Su Antonio Labriola», Nuovo Corriere, 7 febbraio 1954; entrò in campo anche TOGLIATTI, «Per una giusta comprensione del pensiero di Antonio Labriola», quattro puntate in Rinascita dal n. 2 al n. 5 del 1954, le prime tre accompagnate da un «Profilo biografico e intellettuale» del Cafagna (allora fra i più assidui e lucidi collaboratori della stampa comunista); ma Togliatti si fermò sostanzialmente alle premesse storiche - esempio notevole di 'storiografia di un politico' - e purtroppo non sembra che abbia mai portato a termine la ricerca, come pure avrebbe voluto. 
le, cioè la maturità della classe operaia ad uscire di subalternità, a diventare egemone nel processo di costruzione d'un nuovo Stato. Da Stato a Stato, cioè dal primo al secondo Risorgimento, come impostava subito Berti: "LItalia doveva essere uno Stato": le parole con cui Hegel riassunse il pensiero e l'opera di Machiavelli furono l'ideale cui dedicarono la vita Bertrando e Silvio Spaventa, Francesco De Sanctis...». Ma, concludeva il Berti, Spaventa e De Sanctis avevano diagnosticato il male della società italiana, i democratici come Garibaldi avevano cominciato ad operare per sanarli, però era stato Labriola, pronunciando la parola 'socialismo' e cioè transitando da Hegel a Marx, ad aprire un nuova era nella storia del nostro paese:

Che vi fossero già in Italia molteplici aspetti di socialismo sansimoniano o mazziniano o anarchico conta certamente moltissimo, ma non è il fatto decisivo. Il fatto decisivo è che le forze progessive che il nostro Risorgimento aveva già maturate nella società italiana a un certo momento trovarono un punto di confluenza, ch fu al tempo stesso un punto di trasformazione, di salto dialettico, in quel socialismo marxista che Antonio Labriola riassunse ed elaborò nel suo pensiero.

Valentino Gerratana, fattosi poi altrimenti benemerito come editore critico dei Quaderni gramsciani, toccò di lì a poco uno degli argomenti più spinosi per i crociani e cruciale per i marxisti, il rapporto con Labriola di Croce, ${ }^{27}$ cioè la mediazione labrioliana fra Croce, hegelismo napoletano e marxismo: che era l'unico aggancio possibile per fondare storicamente lo slittamento da Croce a Gramsci (oltre che il processo da Hegel a Marx) e per dare una chiave di lettura progressiva, sulla linea appunto Labriola - [Croce]— Gramsci, alla tradizione nazionale di De Sanctis e degli Spaventa. La valorizzazione del marxismo di Labriola, oltre che a determinare una linea democratico-nazionale, serviva anche a riempire il vuoto di dottrina marxista che aveva caratterizzato «il movimento politico delle classi lavoratrici italiane» nel trentennio precedente la prima guerra mondiale: nel sottolineare questa esigenza, Alicata la faceva coincidere con quella di dare alla cultura italiana, contro l'astrattezza idealistica e il tecnicismo empiristico, una nuova sintesi umanistica fondata sulla ragione storica ${ }^{28}$ ed elencava puntualmente i nomi, oltre che di Garin per la sua comprensione dell'inscindibile nesso in Gramsci fra attività rivoluzionaria e attività culturale), di Labriola e del suo stile, di De Sanctis in quanto «modello di uomo di cultura militante».

Sul nodo Labriola-Gramsci, ad ogni modo, Gerratana tornerà subito dopo (in Rinascita, e insomma era tutta la rete della stampa comunista a venir impe-

27. «Labriola e Croce», Il Contemporaneo, 23 ottobre 1954, p. 3-4).

28. M. Alicata, "Un allievo di Gramsci», Il Contemporaneo, II, 32, 6 agosto 1955, p. 1. L'articolo commemorava Ruggero Grieco, l'appena scomparso ispiratore della politica agraria e meridionalista del PCI, uno degli ideatori di Cronache meridionali, che gli dedicherà un fascicolo speciale (II, 12, dicembre 1955). Grieco, sempre nella preoccupazione delle linee storiche, veniva messo in serie con Giustino Fortunato e Guido Dorso. 
gnata), proprio in un articolo di polemica frontale contro la neonata Nord e Sud e il suo programma essenzialmente antigramsciano: si stava tentando adesso contro Gramsci, secondo Gerratana, la medesima opera di neutralizzazione attuata contro Labriola alla fine dell'Ottocento. Essenziale dunque, anche per contrastare questo disegno, il nesso Croce-Labriola: perché, mentre il Partito Socialista aveva lasciato cadere l'insegnamento labrioliano nel vuoto di teoria fra riformismo e massimalismo, quell'eredità, come nel caso di De Sanctis, l'aveva fatta propria e inaridita l'idealismo crociano. ${ }^{29} \mathrm{E}$ nel «Labriola e Croce» del Contemporaneo Gerratana ripercorreva puntigliosamente tutte le fasi dei nessi Spaventa-Labriola e Labriola-Croce, ${ }^{30}$ mostrando i due percorsi opposti compiuti da Labriola e da Croce, il primo dalla Destra storica di Bertrando Spaventa al marxismo, il secondo dal materialismo storico ad una vera e propria, e progressivamente sempre più accanita, militanza antisocialista; mostrando altresì come a Torino - mentre Croce nel 1911 dichiarava sepolto (da lui medesimo) il socialismo teorico- due giovanissimi studenti entrasssero nuovamente in dimestichezza con Labriola e con la sua teoria marxista (ma leggevano, e come!, anche il Croce degli anni vociani...) e si avviassero a riaprire insieme alla classe operaia torinese un nuovo ciclo marxista e rivoluzionario.

Quanto il controllo del caposaldo Labriola fosse considerato strategico è confermato (oltretutto il cinquantesimo anniversario della morte, nel 1954, offriva un'occasione preziosa) dalla diffusione e autorevolezza degli interventi: l'articolo di Gerratana giungeva infatti dopo un'annata martellante. Aveva cominciato Giuseppe Berti annunciando su L'Unità del 13 gennaio che, per sua medesima cura, Rinascita avrebbe pubblicato un fascio di ben 123 lettere inedite indirizzate fra 1870 e 1883 dal Labriola a Bertrando Spaventa: ${ }^{31}$ Rinascita, si badi bene, la allora mensile 'rassegna di politica e di cultura italiana' particolarmente indirizzata alla formazione e all'orientamento del quadro politico attivo, non i più scientifici periodici d'area quali Società o Movimento

29. "L’opera di Gramsci nella cultura italiana», Rinascita, novembre-dicembre 1954, p. 74953. Subito di seguito Mario SPINELLA («Presenza del marxismo», p. 753-56) rivendicava l'efficacia dell'azione marxista nella cultura e in particolare nel campo dell'estetica, dove si stava sbloccando "una situazione particolarmente chiusa a causa del monopolio crociano", e ciò in particolare, si noti, attraverso «il ripristino della figura e dell'importanza decisiva degli scritti e soprattutto del pensiero di Francesco De Sanctis» (un curiosità: il ritratto di Labriola campeggiava, invece che nel corpo dell'articolo labrioliano di Berti, di fianco a questa apologia desanctisiana).

30. Tuttavia il profilo di Labriola più ampio fu quello, affidato alla sede mensile di Rinascita e nella compagnia prestigiosa di Togliatti, compilato in tre puntate da Luciano Cafagna, gia ricordato.

31. Le lettere furono pubblicate dal Berti (ideologicamente usava spesso l'accetta, ma era un paziente, abile, affidabilissimo ricercatore di documenti, e nel complesso un ottimo storicopolitico) nei supplementi di Rinascita del dicembre 1953 e del gennaio 1954. Lo stesso Berti, nel supplemento del marzo 1954, pubblicò altre lettere del Labriola, fra cui particolarmente notevoli quelle indirizzate a Ruggero Bonghi nel 1872, quando Labriola era ancora schierato a Destra. Va detto che l'introduzione premessa dal Berti alle lettere a Bertrando Spaventa (p. 713-18 del supplemento), nella quale fissò tempi e modi della conversione labrioliana prima al socialismo e poi al marxismo, si leggono ancora utilmente. 
Operaio, come ci si sarebbe potuti aspettare per un evento storiografico di tale portata. Di quell'episodio politico-culturale era dunque l'aspetto della fruizione politica interna ad essere valorizzato ancor prima del messaggio per l'esterno, quasi una questione identitaria; del resto, il taglio dell'annuncio di Berti - una vera e propria chiave di interpretazione fornita ai lettori del quotidiano di partito- era inequivocabile: "Queste lettere [...] ci aiutano a meglio capire da dove è venuto il marxismo italiano». Che voleva dire dalla destra storica al marxismo, nel solco (come già Marx ed Engels in Germania) della tradizione nazionale capace di pensare lo Stato, lasciando perdere «la cronachetta delle conventicole anarcoidi o dei gruppetti più o meno socialisteggianti» (un implicito pollice verso - e ingiusto, ma il senso politico era ben chiaro- al taglio che alla ricerca storica di «Movimento Operaio», il movimento operaio per sé, insisteva a voler conservare per esempio il fondatore di quella rivista Gianni Bosio): "Così nelle grandi correnti di sviluppo del pensiero e della società italiana. [...] così continua ed unifica e porta a un più alto livello la sinistra e la destra storica. Così continua i democratici e al tempo stesso continua Francesco De Sanctis, Bertrando Spaventa e Angelo Camillo De Meis. [...]. Così, più o meno compreso, getta le basi di un partito che egli non vedeva come una conventicola anarchica o un intruglio riformista ma come una forte compagine popolare».

Nel linguaggio diretto del giornale quotidiano il Berti, partendo da Labriola, andava anche oltre Gramsci, arrivava al partito di Togliatti: non è difficile immaginare - un tragitto da Spaventa a Labriola al PCI senza neppur più la mediazione di Croce! - l'allegar di denti dei redattori crociani di Nord e Sud. Ma questo era il terreno su cui (edizioni, ricerca storico-critica, polemiche politiche) si stava impiantando il decennio desanctisiano: cioè, all'interno della complessiva questione risorgimentale, il fronte più caldo della battaglia fra marxisti e idealisti (ma sarà meglio dire fra comunisti e crociani) per l'egemonia storicista. 